\begin{abstract}
We find conditions on the order on subsets of a finite set which are necessary and sufficient for the relative ranking of any two subsets in this order to be determined by their extreme elements relative to an abstract convex geometry. It turns out that this question is closely related to the rationalisability of path independent choice functions by hyper-relations.
\end{abstract}




\title{
Orders on Subsets Rationalised by Abstract Convex Geometries
}

\author{
Walter Bossert, ${ }^{*}$ Matthew J. Ryan ${ }^{\dagger}$ and Arkadii Slinko ${ }^{\ddagger}$
}

April 18, 2008

\section{Introduction}

Let $X$ be a finite set and $\mathcal{A}$ the set of non-empty subsets of $X$. Suppose that elements of $\mathcal{A}$ are interpreted as acts in an environment of uncertainty. Under this interpretation, an act is identified with its set of possible consequences. ${ }^{1}$ Let $\succsim$ be a complete and transitive preference order on $\mathcal{A}$. A classic literature, starting with Kannai and Peleg (1984) and surveyed by Barberà, Bossert and Pattanaik (2004), assumes that the singletons in $\mathcal{A}$ are linearly ordered by $\succsim$ and identifies further conditions on $\succsim$ under which the relative ranking of any two subsets is determined by their "extreme" elements. In particular, defining

$$
\begin{aligned}
& \bar{A}=\{a \in A \mid\{a\} \succ\{b\} \text { for all } b \in A \backslash\{a\}\} \\
& \underline{A}=\{a \in A \mid\{b\} \succ\{a\} \text { for all } b \in A \backslash\{a\}\},
\end{aligned}
$$

Kannai and Peleg present simple axioms that imply $A \sim \bar{A} \cup \underline{A}$ for each $A \in \mathcal{A}$. While Kannai and Peleg's axioms cannot be satisfied when $|X| \geq 6$, subsequent authors have weakened them so that suitable orders exist for any finite $|X|$ while preserving the property that $A \sim \bar{A} \cup \underline{A}$. See Bossert and Slinko (2006) for a recent contribution and related literature.

${ }^{*}$ CIREQ/Département de sciences économiques, Université de Montréal

${ }^{\dagger}$ Department of Economics, University of Auckland

${ }_{\ddagger}^{\ddagger}$ Department of Mathematics, University of Auckland

${ }^{1}$ It is natural to suppose that the acts being compared are not related through statewise dominance. Since we deal with finite sets, for which no particular structure is needed, the assumption that dominated acts have been removed is unproblematic. 
The purpose of this note is to identify axioms under which preferences are determined by "extreme" outcomes for a more general notion of "extremeness". In doing so, we shall illustrate the close connection between this problem and one from a quite different strand of the decision theory literature.

In the next section, we recall the notion of a convex geometry for a discrete set. This algebraic theory of convexity provides a natural and quite general definition of an "extreme point". We wish to identify conditions on $\succsim$ that are necessary and sufficient for the existence of a convex geometry such that the relative ranking of any two subsets is determined by their "extreme" elements with respect to this geometry.

In Section 3, we shall seek a solution to this problem in an unexpected quarter. We there recall some results from the theory of choice functions. For this purpose, we reinterpret the subsets of $X$ as opportunity sets. A choice function is a mapping $C: 2^{X} \rightarrow 2^{X}$ that satisfies $C(A) \subseteq A$ for any $A$ and $C(A)=\emptyset$ if and only if $A=\emptyset$. A choice function $C$ satisfies path independence (Plott, 1973) if

$$
C(A \cup B)=C(C(A) \cup B)
$$

Aizerman and Malishevski (1981) and Nehring (1997) characterise the path independent choice functions in terms of "hyper-relations" on $X$. (We shall define this term precisely in Section 3.) Our main result turns out to be a corollary of the characterisation in Nehring (1997).

The link between these two seemingly unrelated problems is provided by the work of Koshevoy (1999), who showed that there is a one-to-one relationship between the set of extreme point operators for convex geometries and the set of path independent choice functions.

\section{Abstract convex geometries}

The abstract notion of a convex geometry is based on the idea of "closing" a set, in the sense of forming a convex hull. Recall (Edelman and Jamison, 1985) that $\mathcal{L}: 2^{X} \rightarrow 2^{X}$ is a closure operator if it satisfies, for all $A, B \subseteq X$ :

(C1) $A \subseteq \mathcal{L}(A)$

(C2) $A \subseteq B$ implies $\mathcal{L}(A) \subseteq \mathcal{L}(B)$,

(C3) $\mathcal{L}(\mathcal{L}(A))=\mathcal{L}(A)$ 
A closure operator $\mathcal{L}$ is called a convex geometry if it satisfies, in addition, the antiexchange property: for any set $A$ satisfying $A=\mathcal{L}(A)$ and any $a, b \in X \backslash A$ such that $a \neq b$, if $b \in \mathcal{L}(A \cup\{a\})$ then $a \notin \mathcal{L}(A \cup\{b\})$.

If $\mathcal{L}$ is a convex geometry, we may interpret $\mathcal{L}(A)$ as the convex hull of $A$. Sets of the form $\mathcal{L}(A)$ for some $A$ are called convex. Given a convex geometry $\mathcal{L}$, its associated extreme point operator ex: $2^{X} \rightarrow 2^{X}$ is defined as follows:

$$
a \in \operatorname{ex}(A) \quad \text { iff } \quad a \in A \text { and } a \notin \mathcal{L}(A \backslash\{a\}) .
$$

The following theorem will play a central role in proving our main result.

Theorem 1 (Koshevoy, 1999). An extreme point operator for a convex geometry is a path independent choice function, and conversely, any path independent choice function is the extreme point operator for some convex geometry.

Given a complete and transitive preference order $\succsim$ on $\mathcal{A}$, we are interested in finding necessary and sufficient conditions for a ranking of subsets to depend only on its extreme points relative to some convex geometry. To be more precise, define the mappings Int : $2^{X} \rightarrow 2^{X}$ and Ext : $2^{X} \rightarrow 2^{X}$ as follows: $\operatorname{Int}(\emptyset)=\operatorname{Ext}(\emptyset)=\emptyset$ and

$$
\begin{aligned}
& \operatorname{Int}(A)=\{a \in A \mid A \sim A \backslash\{a\}\}, \\
& \operatorname{Ext}(A)=A \backslash \operatorname{Int}(A)
\end{aligned}
$$

for any $A \in \mathcal{A}$. Thus, $\operatorname{Ext}(A)$ contains those members of $A$ whose individual removal would alter the ranking of the subset. We shall identify conditions under which Ext is the extreme point operator for some convex geometry and $A \sim \operatorname{Ext}(A)$ for any $A \in \mathcal{A}$. For example, in the case of preferences satisfying the axioms of Kannai and Peleg,

$$
A \sim \operatorname{Ext}(A)=\underline{A} \cup \bar{A},
$$

and Ext is the extreme point operator for the convex geometry with closure operator: ${ }^{2}$

$$
\mathcal{L}(A)=\{x \in X \mid \bar{A} \succsim\{x\} \succsim \underline{A}\}
$$

\section{Rationalising choice functions}

Aizerman and Malishevski (1981) and Nehring (1997) study choice functions that are rationalised by generalised binary relations (or hyper-relations). A generalised binary

\footnotetext{
${ }^{2}$ The anti-exchange property of this operator is guaranteed by Kannai and Peleg's assumption that $\succsim$ linearly orders the singletons. We do not require this assumption for our general result.
} 
relation $\sqsupset$ is a subset of $\mathcal{A} \times X$. We write $A \sqsupset a$ for $(A, a) \in \sqsupset$, and interpret $A \sqsupset a$ to mean that "choice $a$ is unacceptable in the presence of $A \backslash\{a\}$ ". The generalised binary relation $\sqsupset$ is said to rationalise $e^{3}$ the choice function $C$ if

$$
C(A)=\{a \in A \mid \text { there is no } B \subseteq A \text { such that } B \sqsupset a\}
$$

This generalises the idea of a choice function being rationalised by a binary relation on $X$. To motivate the generalisation, imagine that the elements of $A$ are pure strategies in a given strategic-form game. Suppose the decision-maker regards a strategy as acceptable iff it is not strictly dominated by any mixed strategy that can be formed using the available pure strategies. Then we would specify $A \sqsupset a$ iff there is a mixture over $A$ that strictly dominates $a$, and this generalised binary relation would rationalise her choice function. Note that the choice function may not be rationalisable by any binary relation on $X$, as there may exist some pure strategy in $A$ that is not strictly dominated by any other pure strategy in $A$, yet is strictly dominated by some mixture over $A \backslash\{a\}$. (See also Nehring, 1997, Example 1.)

Following Nehring (1997), ${ }^{4}$ we shall impose the following "irreflexivity" and "monotonicity" conditions on $\sqsupset$ :

$$
\begin{gathered}
A \sqsupset a \quad \Longrightarrow \quad[A \backslash\{a\} \neq \emptyset \text { and } A \backslash\{a\} \sqsupset a], \\
A \sqsupset a \quad \Longrightarrow \quad A \cup\{b\} \sqsupset a
\end{gathered}
$$

for any $b \in X$. A generalised binary relation that satisfies (IRR) and (MON) will be called an extended preference relation (EPR). Note that an EPR $\sqsupset$ rationalises the choice function $C$ iff

$$
C(A)=A \backslash\{a \in A \mid A \sqsupset a\}
$$

for each $A \in \mathcal{A}$. An extended partial order (EPO) is an EPR that satisfies the following "transitivity" condition:

$$
[A \backslash\{a\} \sqsupset a \text { and } A \sqsupset b] \quad \Longrightarrow \quad A \backslash\{a\} \sqsupset b .
$$

This may be re-written as

$$
[A \backslash\{a\} \sqsupset a \text { and }(A \backslash\{a\}) \cup\{a\} \sqsupset b] \quad \Longrightarrow \quad A \backslash\{a\} \sqsupset b
$$

\footnotetext{
${ }^{3}$ The terminology is Nehring's. Aizerman and Malishevski say that $C$ is "generated by a strongly dominant choice mechanism". We have opted for the more "economical" nomenclature!

${ }^{4}$ We state a seemingly weaker version of (MON), but easily seen to be equivalent.
} 
which shows it to be a special case of the Transitivity condition in Nehring (1997, p.410). In fact, it turns out that our (TRA), in the presence of (MON) and (IRR), is equivalent to Nehring's seemingly stronger version of transitivity.

The following result is a variation on Nehring (1997, Theorem 1(ii)). ${ }^{5}$ In particular, path independence may be shown to be equivalent to Nehring's conditions $(\alpha)$ and $(\eta){ }^{6}$ We provide a direct proof here for completeness.

Theorem 2. If $\sqsupset$ is an EPO, then (2) is a path independent choice function. Conversely, if $C$ is a path independent choice function, then the generalised binary relation $\sqsupset$ defined by

$$
A \sqsupset a \Longleftrightarrow a \notin C(A \cup\{a\})
$$

is an EPO.

Proof. Suppose $\sqsupset$ satisfies (IRR), (MON) and (TRA), and let $C$ be defined by (2). It is obvious that $C(A) \subseteq A$ for any $A \subseteq X$. To see that $C(A) \neq \emptyset$ when $A \neq \emptyset$, suppose to the contrary that $C(A)=\emptyset$ for some non-empty $A=\left\{a_{1}, \ldots, a_{k}\right\} \subseteq X$. Then $A \sqsupset a_{i}$ for each $i \in\{1, \ldots, k\}$. Applying (TRA) successively, we see that

$$
\begin{gathered}
A \backslash\left\{a_{1}\right\} \sqsupset a_{i} \quad i \in\{2, \ldots, k\}, \\
A \backslash\left\{a_{1}, a_{2}\right\} \sqsupset a_{i} \quad i \in\{3, \ldots, k\}, \\
\ldots \\
\left\{a_{k}\right\} \sqsupset a_{k} .
\end{gathered}
$$

which contradicts (IRR). It remains to show that $C$ is path independent. For this, it suffices to verify the Heritage $(H)$ and Outcast $(O)$ conditions (Aizerman and Malishevski, 1981, Theorems 3 and 4; Danilov and Koshevoy, 2005, Proposition 1):

$$
\begin{gathered}
{[a \in C(A) \text { and } a \in B \subseteq A] \Longrightarrow a \in C(B),} \\
a \notin C(A) \Longrightarrow C(A \backslash\{a\})=C(A) .
\end{gathered}
$$

Axiom (MON) implies that $C$ satisfies $(\mathrm{H})$. Let us prove $(\mathrm{O})$. Since $(\mathrm{O})$ is trivial when $a \notin A$, suppose $a \in A$ and that $A \sqsupset a$. For any $b \in A \backslash\{a\}$,

$$
A \backslash\{a\} \sqsupset b \quad \Longrightarrow \quad A \sqsupset b
$$

\footnotetext{
${ }^{5}$ The equivalence of (i) and (ii) in Nehring (1997, Theorem 2) is also closely related.

${ }^{6}$ Since Nehring does not include the condition $C(A)=\emptyset$ iff $A=\emptyset$ in the definition of a choice function, the first part of our Theorem 2 strengthens Nehring's version. For the second part, our argument does not require the full force of this additional property of choice functions - Nehring's assumption $\left(\omega_{1}\right)$ suffices.
} 
by (MON). This shows $C(A \backslash\{a\}) \supseteq C(A)$. The converse we note that $A \backslash\{a\} \sqsupset a$ by (IRR) and by (TRA) we get

$$
A \sqsupset b \quad \Longrightarrow \quad A \backslash\{a\} \sqsupset b
$$

which shows $C(A \backslash\{a\}) \subseteq C(A)$. So $C$ satisfies (O). This proves the first part of the theorem.

For the converse, suppose that $C$ is a path independent function and $\sqsupset$ is defined as in (3). If $A \sqsupset a$, then we must have $A \backslash\{a\} \neq \emptyset$ since $a \notin C(\{a\})$ is excluded by the facts that $C(B) \subseteq B$ for any $B \subseteq X$ and $C(B)=\emptyset$ iff $B=\emptyset$. Also, since $A \cup\{a\}=(A \backslash\{a\}) \cup\{a\}, A \sqsupset a$ implies $A \backslash\{a\} \sqsupset a$. This proves (IRR). Next, suppose that $a \in C(A \cup\{a, b\})$. Then, by path independence, ${ }^{7}$

$$
a \in C(C(A \cup\{a\}) \cup\{b\}) .
$$

Since $C(C(A \cup\{a\}) \cup\{b\}) \subseteq C(A \cup\{a\}) \cup\{b\}$, it follows that $a \in C(A \cup\{a\})$. This proves (MON). Finally, suppose that $A \backslash\{a\} \sqsupset a$ and $A \sqsupset b$. Since we already established that $\sqsupset$ satisfies (MON), these imply

$$
A \cup\{a, b\} \sqsupset a \text { and } A \cup\{a, b\} \sqsupset b .
$$

Equivalently

$$
a \notin C(A \cup\{a, b\}) \text { and } b \notin C(A \cup\{a, b\}) .
$$

Applying condition $(\mathrm{O})$, which is a consequence of path independence, we have:

$$
C(A \cup\{a, b\})=C((A \backslash\{a\}) \cup\{b\})
$$

The latter equality and $C(B) \subseteq B$ for any $B \subseteq X$ imply

$$
b \notin C((A \backslash\{a\}) \cup\{b\}),
$$

which is equivalent to $A \backslash\{a\} \sqsupset b$. This proves (TRA).

\subsection{The main result}

We now return to the problem described at the end of Section 2. Consider the following two Axioms, which are assumed to hold for all $A \in \mathcal{A}$ and all $a, b \in A$ :

Axiom 1 (A1). If $A \sim A \backslash\{a\}$ and $A \sim A \backslash\{b\}$, then $A \sim A \backslash\{a, b\}$.

\footnotetext{
${ }^{7}$ Indeed, as Nehring (1997, Fact 1) points out, the Heritage condition (H) suffices.
} 
Axiom 2 (A2). If $A \sim A \backslash\{a\}$, then $A \cup\{c\} \sim(A \backslash\{a\}) \cup\{c\}$ for any $c \in X$.

We may now state the main result of the paper:

Theorem 3. The following are equivalent:

I. Axioms (A1) and (A2) hold.

II. The function Ext derived from $\succsim$ is the extreme point operator for some convex geometry and $A \sim \operatorname{Ext}(A)$ for any $A \in \mathcal{A}$.

To see the connection between Theorems 2 and 3, suppose that we are given a complete and transitive preference relation $\succsim$ on $\mathcal{A}$. First extend $\succsim$ to $2^{X}$ by specifying $\emptyset \sim \emptyset$ and $A \succ \emptyset$ for any $A \in \mathcal{A}$, and then define the generalised binary relation $\sqsupset$ as follows:

$$
A \sqsupset a \Longleftrightarrow A \cup\{a\} \sim A \backslash\{a\}
$$

It is straightforward to observe that (IRR) is satisfied, while (MON) is equivalent to (A2) and (TRA) is equivalent to (A1). Moreover, if $\sqsupset$ is an EPR and $C$ is the choice function rationalised by $\sqsupset$, then

$$
C(A)=A \backslash\{a \in A \mid a \in \operatorname{Int}(A)\}=\operatorname{Ext}(A) .
$$

Thus, the $(\mathrm{I}) \Longrightarrow(\mathrm{II})$ part of Theorem 3, apart from the claim that $A \sim \operatorname{Ext}(A)$ for all $A \in \mathcal{A}$, follows directly from Theorem 1 and the first part of Theorem 2 .

Lemma 1. Under (A1) and (A2), if $\operatorname{Ext}(A) \subseteq B \subseteq A$, then $A \sim B$ and $\operatorname{Ext}(A)=$ $\operatorname{Ext}(B)$. In particular, $A \sim \operatorname{Ext}(A)$ for every $A \in \mathcal{A}$.

Proof. Note that (A1) and (A2) together give:

$$
a \in \operatorname{Int}(A) \Longrightarrow[b \in \operatorname{Int}(A) \text { iff } A \sim A \backslash\{a, b\}]
$$

Let $\operatorname{Int}(A)=\left\{a_{1}, a_{2}, \ldots, a_{k}\right\}$ with $B=\operatorname{Ext}(A) \cup\left\{a_{i}, a_{i+1}, \ldots, a_{k}\right\}$ for some $i \in\{1,2, \ldots, k-1\}$. It follows from (5) that $\operatorname{Int}\left(A \backslash\left\{a_{1}\right\}\right)=\left\{a_{2}, a_{3}, \ldots, a_{k}\right\}$. Proceding by induction we obtain the desired result.

Conversely, suppose that Ext is the extreme point operator for some convex geometry. Hence, it is a path independent choice function (Theorem 1). Since the generalised binary relation defined by (4) satisfies $A \sqsupset a$ iff $a \notin \operatorname{Ext}(A \cup\{a\})$, it follows from the second part of Theorem 2 that $\sqsupset$ is an EPO and hence that $\succsim$ satisfies (A1) and (A2). This proves the $(\mathrm{II}) \Longrightarrow(\mathrm{I})$ part of Theorem 3 . 


\section{References}

Aizerman M.A. And A.V. Malishevski (1981) "General theory of best variants choice: some aspects," IEEE Transactions on Automatic Control 26, 1030-1041.

Barbera, S., Bossert, W. And P.K. Pattanaik (2004), "Ranking sets of objects," in Barbera, Hammond and Seidl (Eds.), The Handbook of Utility Theory, Volume 2 (Chapter 17). Springer.

Bossert, W. And A. Slinko (2006) "Relative Uncertainty Aversion and Additively Representable Set Rankings," International Journal of Economic Theory 2, 105-122.

Danilov, V.I. And G.A. Koshevoy (2005), "Mathematics of Plott choice functions," Mathematical Social Sciences 49, 245-272.

Edelman, P.H. And R.E. Jamison (1985), "The theory of convex geometries," Geometriae Dedicata 19, 247-270.

KANNAi AND Peleg (1984) "A note on the extension of an order on a set to the power set," Journal of Economic Theory 32, 172-175.

Koshevoy, G.A. (1999), "Choice functions and abstract convex geometries," Mathematical Social Sciences 38, 35-44.

Plott C.R. (1973) "Path independence, rationality and social choice," Econometrica 41, 1075-1091. 